\title{
Contamination des eaux de puits traditionnels par les nitrates sur le bassin versant de la Lobo (Buyo, sud- ouest de la Côte d'Ivoire)
}

\author{
Ohou-Yao M. J. A. ${ }^{1}$, Séka A. M. ${ }^{2}$, Mambo V. ${ }^{2}$, Yapo O. B. ${ }^{2}$,Konan K. F. ${ }^{1}$ Houénou P. V. ${ }^{3}$ \\ 1 UFR - Environnement, Université Jean Lorougnon Guédé Daloa, Côte d'Ivoire ; ${ }^{2}$ Laboratoire des Sciences de \\ l'Environnement, UFR - Sciences et Gestion de l'Environnement, Université Nangui Abrogoua, Abidjan, Côte \\ d'Ivoire ; ${ }^{3}$ Agence Universitaire de la Francophonie (Bureau Afrique de l'Ouest) \\ Auteur correspondant : Ohou-Yao M. J. A. ; BP 150 Daloa, Côte d'lvoire ; GSM : 0022505034532 : \\ ohoujeanne@yahoo.fr
}

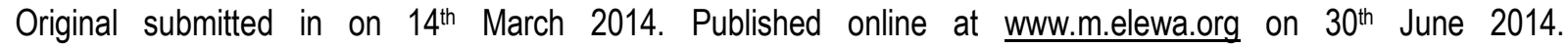
http://dx.doi.org/10.4314/jab.v78i1.11

\section{RÉSUMÉ}

Objectif : La contamination des eaux souterraines par les nitrates est un problème réel de santé publique qui fait actuellement l'objet d'une attention particulière dans le monde entier. Cette étude examine la répartition spatiale des nitrates dans les eaux de puits traditionnels du bassin versant de la Lobo, afin d'en tirer les mesures appropriées pour protéger les ressources en eau souterraine de cette région.

Méthodologie et résultats : l'origine des nitrates des eaux de puits traditionnels du bassin versant de la Lobo a été déterminée à travers l'analyse des concentrations en nitrate. Les échantillonnages ont été effectués en mars et juin 2007 sur les eaux de puits traditionnels dans deux villages Gbili et Logbozoa et des campements sur le bassin versant de la Lobo. Les résultats révèlent que les nitrates subissent une variation sur le bassin versant. Dans l'ensemble, les concentrations vont de $0 \mathrm{mg} \cdot \mathrm{L}^{-1}$ à $71,26 \mathrm{mg} \cdot \mathrm{L}^{-1}$. Les puits ayant une concentration supérieure à la valeur guide de l'OMS (50 mg. $\left.\mathrm{L}^{-1}\right)$ se rencontrent dans la ville de Buyo et dans les deux villages Gbili et Logbozoa. Par contre, les puits de Tchemasso, de Buyo provisoire et des campements ont des teneurs en nitrate conformes à cette norme.

Conclusion et application des résultats : Les puits de la ville de Buyo et des deux villages Gbili et Logbozoa ayant des densités de population plus élevées, ont des eaux plus riches en nitrate. Par contre, les puits de Tchemasso, de Buyo provisoire et des campements, de densité de population plus faible et parfois avec des puits situés dans des plantations ont de faible teneurs. Ainsi, la forte densité de population, associée au défaut d'assainissement et aux latrines mal installées compte tenu de l'exigüité des habitations, constitue la principale cause des teneurs élevées de nitrate dans les puits traditionnels du bassin versant de la Lobo. Les puits situés dans les plantations, avec des eaux ayant de faibles teneurs en nitrate, montrent que l'agriculture n'est pas responsable de la contamination des eaux dans cette zone.

A court terme, une campagne de sensibilisation bien conçue sur l'assainissement et la santé publique devrait être initiée à l'endroit des populations pour permettre d'améliorer et de protéger la qualité de l'eau des puits traditionnels dans le bassin versant de la Lobo.

Mots clés : activité anthropique, eau souterraine, pollution, qualité physico-chimique. 


\section{Abstract}

Objective: Contamination of groundwater by nitrates is a real public health concern that is currently being paid a particular attention worldwide. This study investigates the spatial distribution of nitrates in traditional water supply wells in the Lobo's watershed, in order to derive appropriate measures to protect groundwater resources in this region.

Methodology and Results: the origin of nitrate water from traditional wells of Lobo's watershed was determined through the analysis of nitrate concentrations. The samples were collected in March (dry season) and June (wet season) 2007 on the waters of traditional wells in two villages Gbili and Logbozoa and camps on the lobo's watershed. The results show that nitrate concentrations vary on the watershed. In general, concentrations range from $0 \mathrm{mg} \cdot \mathrm{L}^{-1}$ to $71,26 \mathrm{mg} \mathrm{L}^{-1}$. Wells with a concentration above the guideline value of WHO (50 mg.L-1) are found in Buyo and both villages Gbili and Logbozoa. On the other hand, wells of Tchemasso, Buyo Provisoire and camps have nitrate levels complying with the standard.

Conclusion and application of results: Well waters of Buyo and two villages Gbili and Logbozoa with higher population densities are richer in nitrate. On the contrary, wells of Tchemasso, Buyo Provisoire and camps, with lower population density and sometimes with wells in plantations have low levels. Thus, the high population density, coupled with the lack of sanitation and incorrectly installed latrines, is the main cause of high nitrate levels in traditional water supply wells in the Lobo's Watershed. Water wells located in plantations with low nitrate levels show that agriculture is not responsible for the contamination of water in this area. In the short term, an appropriately designed awareness campaign on sanitation and public health directed to the populations should be initiated to allow improving and protecting well water quality in the Lobo's watershed.

Keywords: anthropogenic activity, groundwater, pollution, physico-chemical quality.

\section{INTRODUCTION}

L'accès à l'eau potable et à l'assainissement est un défi quotidien pour des centaines de milliers de citadins qui vivent principalement dans les pays en développement (Gnagne et al 2006; Yapo et al 2010; Konan et al 2011). En milieu rural, cette difficulté se traduit par l'insuffisance des ouvrages améliorés pour l'approvisionnement en eau potable et pour l'assainissement, ainsi que par des pannes répétées. Buyo, ville située au sud-ouest de la Côte d'Ivoire n'échappe pas à cette difficulté. Ce qui amène les populations à se tourner vers les eaux de puits traditionnels qui représentent, non pas une source alternative, mais la principale source d'approvisionnement en eau potable. Des études sur la qualité physico-chimique des eaux souterraines en Côte d'Ivoire ont été réalisées par Faillat (1990), plus précisément celles sur la pollution en nitrate conduites par Savané et al (2005). Cependant, peu d'études ont été réalisées dans la région de Buyo (Yapo et al 2008 ; Mambo et al 2001). Ces études se sont surtout intéressées aux eaux de surface. Très peu de travaux (Ohou et al 2008) ont porté sur la qualité des eaux de puits traditionnels. Cette étude a mis en évidence la pollution en nitrate des eaux de puits traditionnels dans la ville de Buyo et deux villages environnants, pollution domestique due à la densité de la population. Cependant, la région étant à vocation agricole, l'agriculture a souvent été indexée. La présente étude qui s'inscrit à la suite de ces travaux se propose d'une part de caractériser la qualité physico-chimique des eaux de puits à usage domestique sur le bassin versant de la Lobo (dans la région de Buyo), et d'autre part, d'identifier l'origine (domestique ou agricole) de cette pollution et d'évaluer son impact sur la qualité des eaux. 


\section{MATÉRIEL ET MÉTHODES}

Présentation de la zone d'étude : La sous-préfecture de Buyo est localisée à l'ouest de la Côte d'Ivoire entre les latitudes $6^{\circ} 10^{\prime} \mathrm{N}$ et $7^{\circ} \mathrm{N}$ et les longitudes $7^{\circ} 30^{\prime} \mathrm{W}$ et $6^{\circ} 50^{\prime} \mathrm{W}$. Elle est située au Nord du département de
Soubré, et est délimitée par les sous-préfectures de Guiglo, Taï et Duekoué à l'Ouest, Issia au Nord, GrandZattry à l'Est et Méadji au Sud (figure 1).

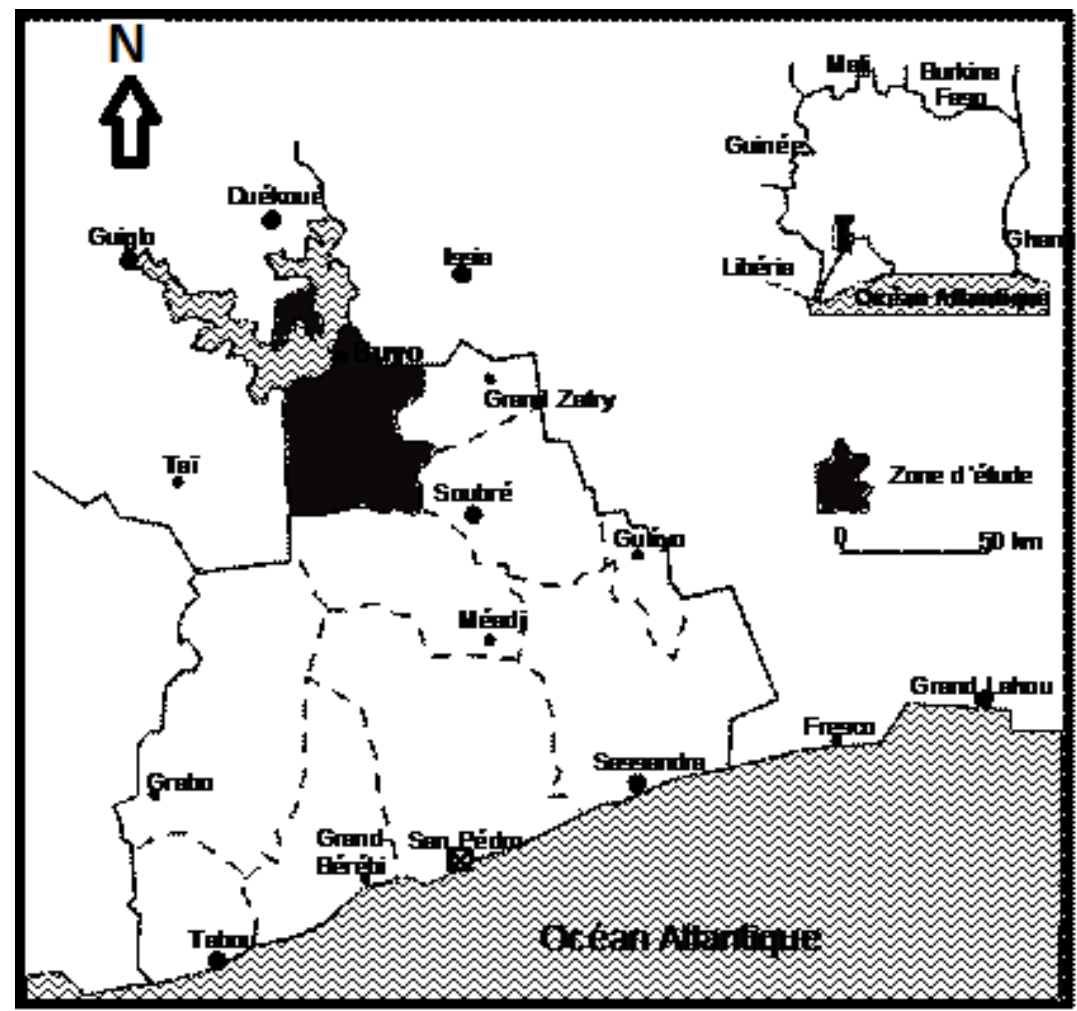

Figure 1 : Localisation de la sous-préfecture de Buyo

L'étude a été réalisée sur le bassin versant de la rivière Lobo (figure 2), un des sous bassins du fleuve Sassandra, situé entre les latitudes $6^{\circ} 18^{\prime} \mathrm{N}$ et $6^{\circ} 37^{\prime} \mathrm{N}$ et les longitudes $6^{\circ} 85^{\prime} \mathrm{W}$ et $7^{\circ} 02^{\prime} \mathrm{W}$. Elle a pris en compte la ville de Buyo, deux villages Gbili (G) et Logbozoa (L) et des campements. Ces localités ont été choisies en tenant compte des activités qui s'y déroulent, de la densité de la population et des points d'approvisionnement en eau potable disponibles. 


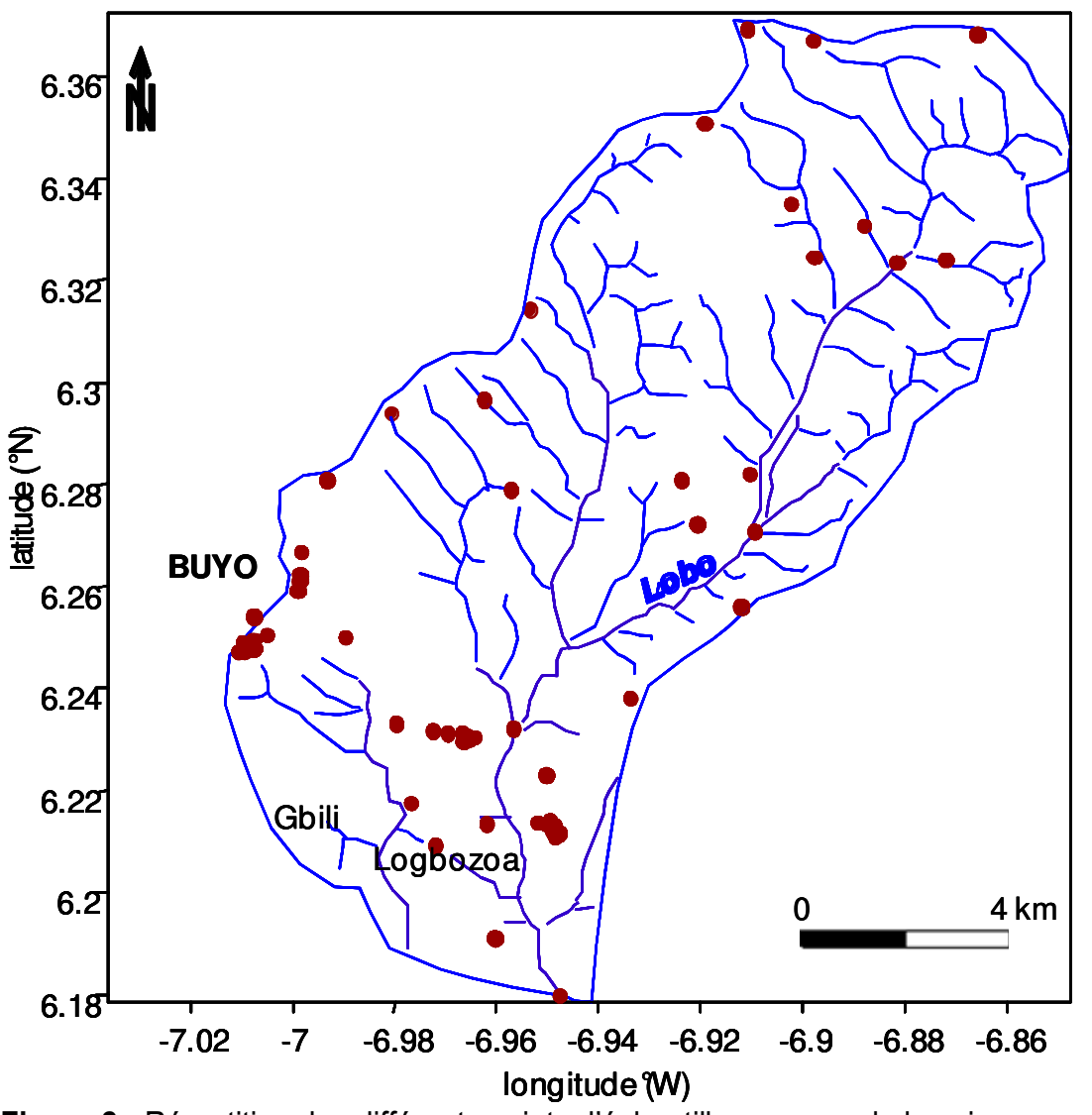

Figure 2 : Répartition des différents points d'échantillonnage sur le bassin versant de la Lobo

La ville de Buyo se trouve sur un site présentant un relief de plateaux dont les pentes sont relativement faibles, avec toutefois la présence de nombreux basfonds et cours d'eau. Cette ville s'est développée à la suite de la construction du barrage hydroélectrique qui porte son nom. Cette cité a été créée conjointement sur deux plateaux distants l'un de l'autre de deux (2) kilomètres. Ce sont: le plateau sud qui représente le noyau d'origine de la ville ; il abrite une cité des cadres du barrage dénommée "Cité $\mathrm{EECl}$ » et le plateau nord qui abrite la cité des ouvriers et autres agents, dénommée "Nouveau Buyo ». Elle bénéficie d'un réseau d'adduction d'eau potable alimenté à partir de la retenue d'eau du barrage. Au niveau du drainage, l'écoulement des eaux de pluie se fait naturellement grâce à la topographie du site. Les eaux de pluie sont directement déversées dans les bas-fonds ou dans la retenue d'eau. Cette ville est composée de neuf (9) quartiers. Pour l'assainissement de la ville, seul le nouveau quartier dispose d'un réseau de collecte des eaux usées qui demande toutefois une remise en état. L'étude s'est déroulée dans trois quartiers de la ville que sont Buyo cité, Quartier Lac (communément appelé Buyo provisoire) et Tchemasso. Buyo cité est composé d'habitats traditionnels de type spontané, et abrite l'actuel marché. Ce quartier est construit en matériaux précaires et renferme plus de $55 \%$ de la population de la ville. Buyo provisoire et Tchemasso sont composés d'habitats économiques et évolutifs. Ils se localisent à l'entrée Est de la ville en provenance d'Issia et regroupent $30 \%$ de la population de la ville. Dans l'ensemble, tous les ménages des quartiers de la ville disposent de latrines. Toutefois, la population des quartiers précaires et surtout les anciennes habitations ne disposent pas de latrines. Dans les deux derniers cas, les eaux usées sont rejetées dans la nature, dans les cours, aux abords des rues. Ce qui crée une humidité constante dans ces quartiers. Les habitants de ces quartiers ne disposant pas de latrines, utilisent la nature et les broussailles environnantes comme lieu d'aisance. Les villages Gbili et Logbozoa sont situés à moins de $10 \mathrm{~km}$ de la ville et sont distants de $2 \mathrm{~km}$. Ils ne disposent pas de réseau d'adduction d'eau potable. Les eaux de puits représentent leur principale source d'alimentation en eau potable. Les puits sont situés dans les cours. La densité de population est 


\section{Ohou-Yao et al. J. Appl. Biosci. 2014. Contamination des eaux de puits traditionnels par les nitrates}

sur le basin versant de la Lobo, Cote d'Ivoire

relativement faible par rapport à celle de la ville. Les campements sont dispersés sur le bassin versant de la rivière. Comme les villages, ils ne disposent pas de réseau d'adduction en eau potable. Les puits sont également situés dans les cours. Cependant, à la différence des autres localités, les puits se retrouvent parfois en pleine plantation (cacao, café). La densité de population est faible.

Prélèvement et mesure des paramètres physicochimiques: L'échantillonnage a couvert les saisons sèche et humide. Les paramètres physiques tels que la température, le $\mathrm{pH}$, et la conductivité ont été mesurés in situ à chaque point d'eau échantillonné. Pour ces différents paramètres, les appareils de mesure préalablement calibrés sont mis sous tension quelques minutes avant toute manipulation. Les sondes sont plongées ensuite dans l'échantillon d'eau prélevé et la sélection de la fonction du paramètre désiré donne la valeur de ce dernier sur l'écran d'affichage. Les échantillons d'eau sont collectés dans des bouteilles en polyéthylène de 1 litre (Pacheco et al., 2001) en fonction de l'élément à analyser. Chaque flacon est rincé et rempli avec l'eau prélevée. Les échantillons destinés à l'analyse des ions $\mathrm{Ca}^{2+}, \mathrm{Mg}^{2+}, \mathrm{Na}^{+}$et $\mathrm{K}^{+}$sont acidifiés par ajout de $0,5 \mathrm{ml}$ d'acide sulfurique concentré dans $250 \mathrm{ml}$ d'eau. Pour l'analyse des ions $\mathrm{NH}_{4}^{+}, \mathrm{NO}_{2}{ }^{-}, \mathrm{NO}_{3}{ }^{-}$, $\mathrm{HCO}_{3}$; les échantillons ne subissent aucun prétraitement. Tous ces flacons sont conservés dans une glacière et acheminés au laboratoire, où ils sont stockés à une température de $-5^{\circ} \mathrm{C}$.

Analyses chimiques: Les analyses ont porté sur les ions $\mathrm{Ca}^{2+}, \mathrm{Mg}^{2+}, \mathrm{Na}^{+}, \mathrm{K}^{+}, \mathrm{NH}_{4}{ }^{+}, \mathrm{NO}_{2}-, \mathrm{NO}_{3}{ }^{-}, \mathrm{HCO}_{3}$. Les ions sodium et potassium ont été dosés par spectrophotométrie d'émission de flamme. Les teneurs en ions $\mathrm{Ca}^{2+}$ et $\mathrm{Mg}^{2+}$ sont obtenues après détermination du titre hydrotimétrique $(\mathrm{TH})$ et du titre hydrotimétrique total (THT). La méthode utilisée pour le dosage de l'ammonium est celle au bleu d'indophénol. La détermination du nitrite s'est faite par la diazotation, et la méthode au salicylate a été utilisée pour le nitrate.

\section{RÉSULTATS}

Caractérisation physico-chimique des eaux: La variabilité spatiale des paramètres physico-chimiques a été étudiée à l'aide du test d'hypothèse non paramétrique de Kruskal Wallis (Siegel, 1956 ; Harris et al 1987), et celui de Mann Witney pour les comparaisons inter et intra sujets. II est réalisé sur les données classées, et a permis de vérifier l'appartenance ou pas des échantillons à une même population, eu égard aux moyennes, au seuil classique
L'analyse des ions bicarbonates s'est effectuée par titrimétrie.

Analyses statistiques : L'utilisation de deux tests nonparamétriques a permis de faire les comparaisons inter et intra des paramètres de la qualité des eaux des puits dans la zone d'étude. Ainsi, le test de Kruskal-Wallis a été utilisé à un seuil de significativité de $95 \%(p<0,05)$, pour mettre en évidence les éventuelles variations entre les différents paramètres de qualité des eaux de puits à l'intérieur des localités. Ensuite lorsque ce test est significatif, l'analyse est poursuivie avec le test de Mann-Whitney, utilisé également pour un seuil de significativité de $95 \%(p<0,05)$. Ce test permet d'étudier la variabilité inter-localité de la qualité des eaux des puits. Ainsi, les localités (représentées en trois catégories : campement, village et ville) ont été comparées deux à- deux, et l'évolution spatiale de chaque paramètre a été étudiée à l'aide des boîtes à moustaches ou "boxplot" (Tomassone et al 1993).

L'algorithme des cartes auto-organisatrices a été utilisé pour ordonner les sites d'études en fonction des variables environnementales. Cet algorithme ou Self Organizing Maps (SOM) encore appelé cartes de Kohonen (Kohonen, 1982 ; Kohonen 1995) appartient au groupe de réseaux de neurones artificiels. Ces derniers sont des modèles mathématiques qui partagent avec les algorithmes classiques d'ordination, l'idée de représenter des données multidimensionnelles dans un espace de dimension inférieure (généralement un plan). Contrairement aux méthodes classiques, les SOM traitent avec beaucoup d'aisance les données extrêmes (abondance ou rareté), très souvent contenues dans les bases de données écologiques (Brosse et al 1999a, b et c; Brosse et al 2001; Giraudel et Lek, 2001 ; Lek et al 1996 et 2000 ; Laë et al 1999 ; Tudesque et al 2008). Ces auteurs ont montré l'efficacité des réseaux de neurones en écologie. D'autres auteurs l'ont utilisé en médecine, en physique et en chimie par exemple pour l'évaluation de la qualité des eaux côtières (Gevrey, 2003).

de $5 \%$. Les résultats de ces tests ont permis de construire les Figures 3,4 et 5 qui décrivent les variations spatiales des paramètres physico-chimiques de qualité des eaux de puits à l'intérieur de chacun des trois types de localités (campement, ville et village).

Le test de Kruskal Wallis réalisé indique qu'il n'y a pas de différence significative dans la variation des ions bicarbonates, calcium, magnésium, potassium, nitrite et le $\mathrm{pH}$ pour la caractérisation de la qualité des eaux de 
puits des localités étudiées. Par contre, il existe des différences statistiquement significatives entre la conductivité, les ions sodium, ammonium et la dureté de l'eau pour lesquelles $p<0,05$. En effet, le test de Mann-Whitney combiné aux boîtes à moustaches, révèle que les valeurs de la conductivité (figure 3 (c)), des ions sodium (figure 4 (a)) et ammonium (figure 5 (b)) sont plus élevées dans les eaux de puits de la ville que celles des campements et des villages.

Température, conductivité et $\mathrm{pH}$ : La température $\left(\mathrm{T}^{\circ} \mathrm{C}\right)$, la conductivité (CND) et le pH mesurés in-situ au moment du prélèvement des échantillons, sont liés aux caractères physiques du milieu. La température montre une certaine homogénéité. Elle varie de $25^{\circ} \mathrm{C}$ à $28,7^{\circ} \mathrm{C}$ avec une moyenne de $26,98( \pm 0,81){ }^{\circ} \mathrm{C}$ pour l'ensemble des eaux de puits (Figure 3 ). Les pH varient de 4,0 à 5,39 avec une moyenne de $4,52( \pm 0,37)$. Toutes les eaux de la zone d'étude sont acides $(\mathrm{pH}<7)$. Elles ne répondent donc pas aux normes pour l'eau de consommation pour l'eau de consommation selon I'OMS. Les conductivités montrent une grande variabilité de la composition chimique. Elles varient entre un minimum de $20 \mu \mathrm{S} / \mathrm{cm}$ et un maximum de 480 $\mu \mathrm{S} / \mathrm{cm}$. Les teneurs sont plus élevées en ville.
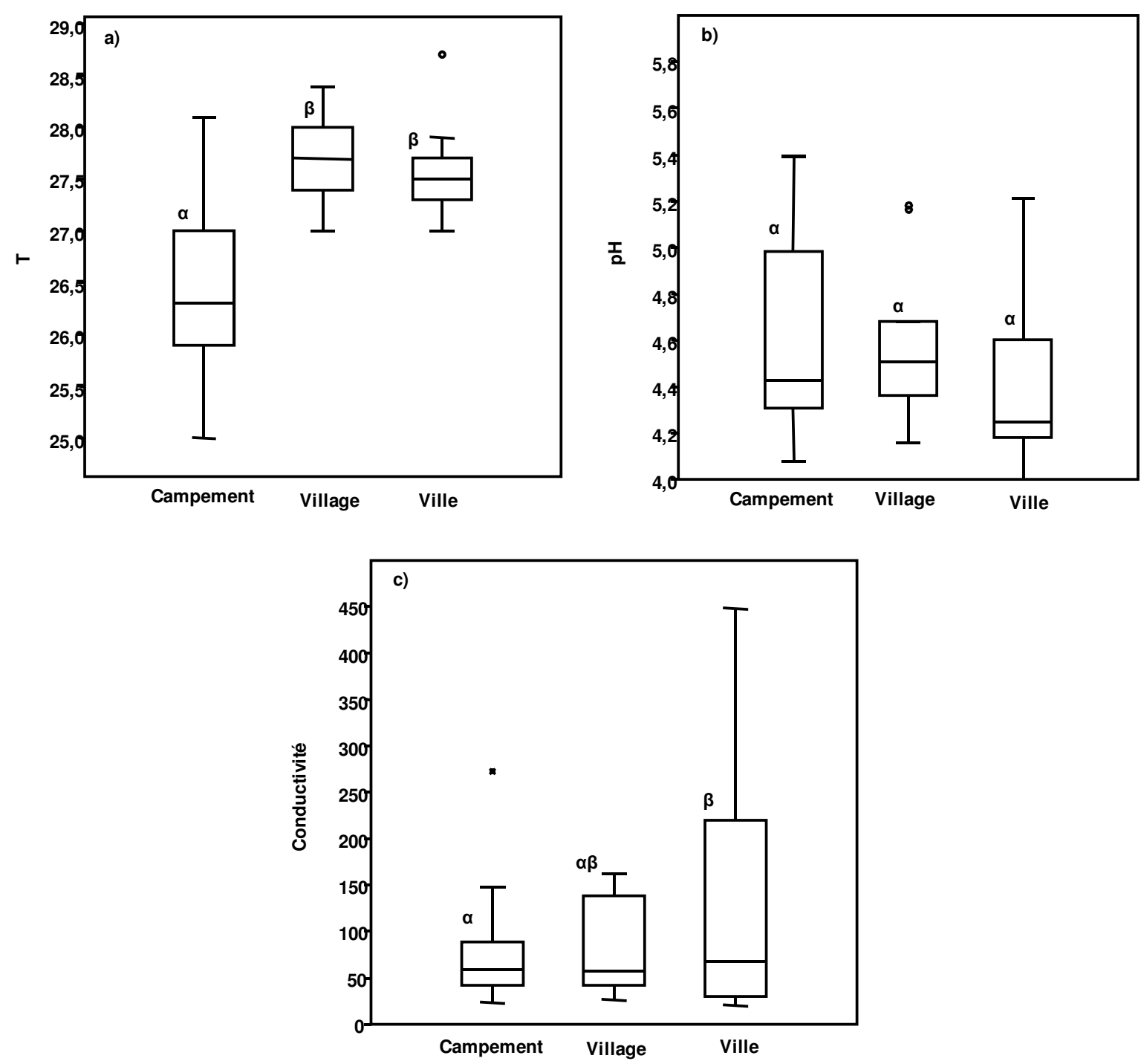

Figure 3: Variabilité de la température (a), du pH (b) et de la conductivité (c) dans les eaux de puits des campements, de la ville et des villages. Pour un paramètre donné, il n'y a pas de différence significative entre deux boîtes portant une même lettre de l'alphabet grec ( $\alpha \beta$ ). Deux lettres différentes traduisent une variabilité importante entre deux points de prélèvement. Le seuil de significativité du test de Mann-Whitney est de $5 \%$. 
Caractérisation chimique des eaux : Les valeurs des coefficients de variation des ions $\mathrm{Na}^{+}, \mathrm{K}^{+}, \mathrm{Ca}^{2+}$ et $\mathrm{Mg}^{2+}$, toutes supérieures à $30 \%$, montrent que ces éléments n'ont pas une répartition homogène dans la zone d'étude. Les teneurs varient pour le $\mathrm{Na}^{+}$de 1,68 à
166,5 avec une moyenne de 19,01 et pour le $\mathrm{K}^{+}$de 0,18 à $51,37 \mathrm{mg} / \mathrm{L}$. Quant au $\mathrm{Ca}^{2+}$, elles vont de 0,08 à $15,2 \mathrm{mg} / \mathrm{L}$ avec une moyenne de 2,2 pendant que celles de $\mathrm{Mg}^{2+}$ oscillent entre 0,024 et $5,66 \mathrm{mg} / \mathrm{L}$ avec une moyenne de 1,54 (figure 4).
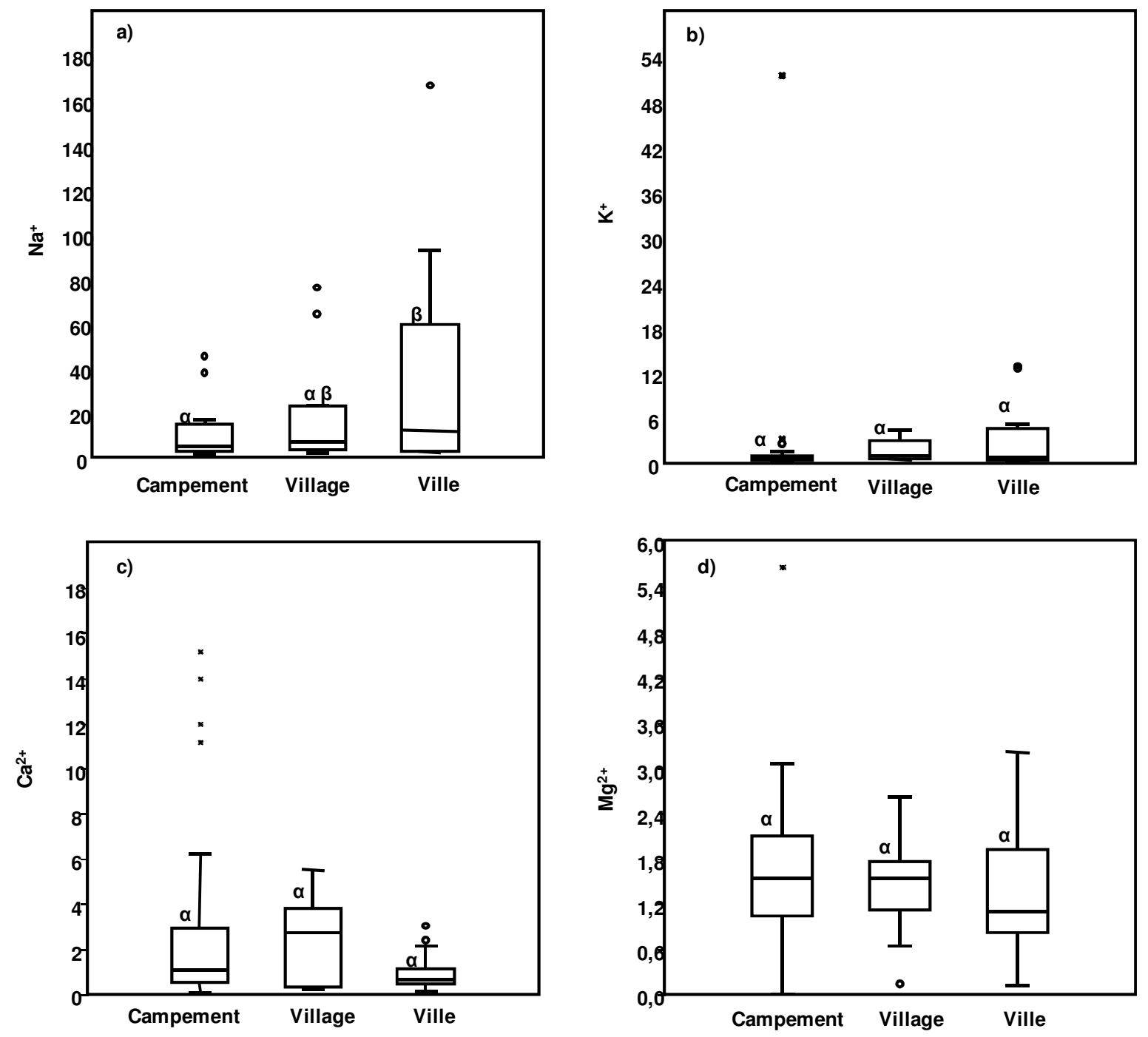

Figure 4: Variation des concentrations en ions a) $\mathrm{Na}^{+}$, b) $\mathrm{K}^{+}$, c) $\mathrm{Ca}^{2+}$ et d) $\mathrm{Mg}^{2+}$, dans les eaux de puits des campements, de la ville et des villages. Pour un paramètre donné, il n'y a pas de différence significative entre deux boîtes portant une même lettre de l'alphabet grec (a $\beta$ ). Deux lettres différentes traduisent une variabilité importante entre deux points de prélèvement. Le seuil de significativité du test de Mann-Whitney est de $5 \%$.

Cette hétérogénéité est également observée avec les ions $\mathrm{HCO}_{3}^{-}, \mathrm{NH}_{4}^{+}, \mathrm{NO}_{2}^{-}, \mathrm{NO}_{3}^{-}$et (figure 5). Ces ions ont des concentrations moyennes respectives de 9,87 $\mathrm{mg} / \mathrm{L}, 0,44 \mathrm{mg} / \mathrm{L}, 0,05 \mathrm{mg} / \mathrm{L}$ et $12,2 \mathrm{mg} / \mathrm{L}$ avec des maxima respectifs de $41,48 \mathrm{mg} / \mathrm{L}, 2,4 \mathrm{mg} / \mathrm{L} 1,3 \mathrm{mg} / \mathrm{L}$ et $71,26 \mathrm{mg} / \mathrm{L}$. 

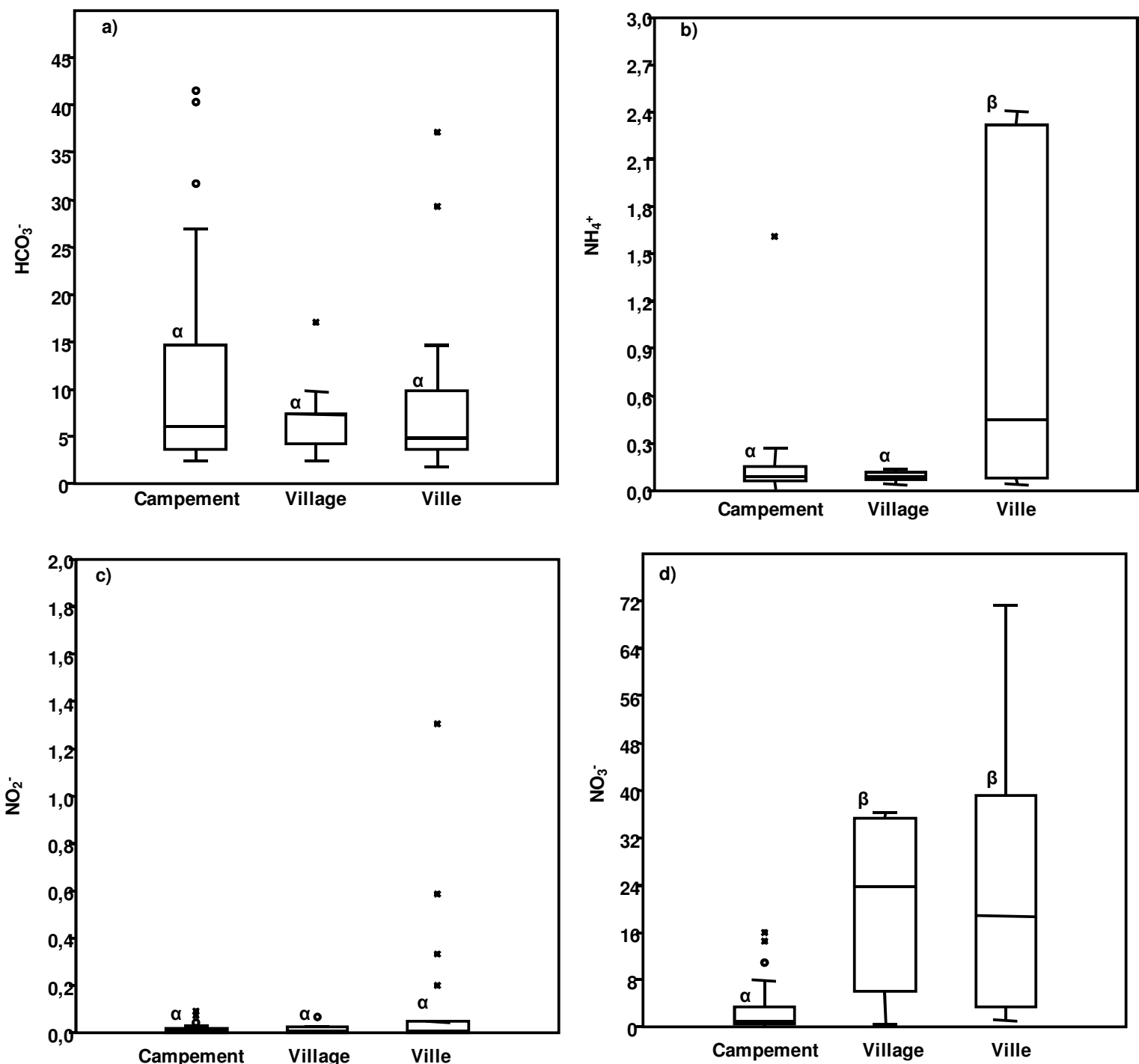

Figure 5 : Variation des concentrations en ions a) $\mathrm{HCO}_{3}^{-}$, b) $\mathrm{NH}_{4}{ }^{+}$, c) $\mathrm{NO}_{2}^{-}$et d) $\mathrm{NO}_{3}^{-}$et dans les eaux de puits des campements, de la ville et des villages. Pour un paramètre donné, il n'y a pas de différence significative entre deux boîtes portant une même lettre de l'alphabet grec $(\alpha \beta)$. Deux lettres différentes traduisent une variabilité importante entre deux points de prélèvement. Le seuil de significativité du test de Mann-Whitney est de $5 \%$.

Malgré cette répartition hétérogène, les teneurs en cations ont des valeurs qui respectent en général les normes de l'OMS pour l'eau de consommation. En ce qui concerne les éléments azotés, les teneurs sont également dans la majorité des cas, inférieures à la norme de l'OMS (50 mg/L). Cependant, c'est en ville que les puits présentent des eaux de teneurs plus élevées en ions azotés.

Variation spatio-temporelle des paramètres physico-chimiques des eaux : L'apprentissage de la SOM a permis de classer les échantillons en fonction de la distribution des paramètres physico-chimiques. Une carte de 35 cellules (7 lignes x 5 colonnes) a été retenue. Les stations sont ensuite réparties sur la carte en fonction des paramètres physico-chimiques qui les discriminent. Ensuite, une analyse de classification hiérarchique nous a permis de regrouper les 35 cellules de la carte en sous-ensembles (I à IV) (figure 6). Ainsi, nous remarquons que le groupe I rassemble en majorité les puits des villages de Gbili et de Logbozoa. Quant au groupe II, il renferme quelques puits des campements, mais en majorité les puits de Tchemasso et de Buyo provisoire. Concernant le groupe III, il se constitue majoritairement des puits de Buyo cité. Enfin, le groupe IV rassemble la majorité des puits des campements. 


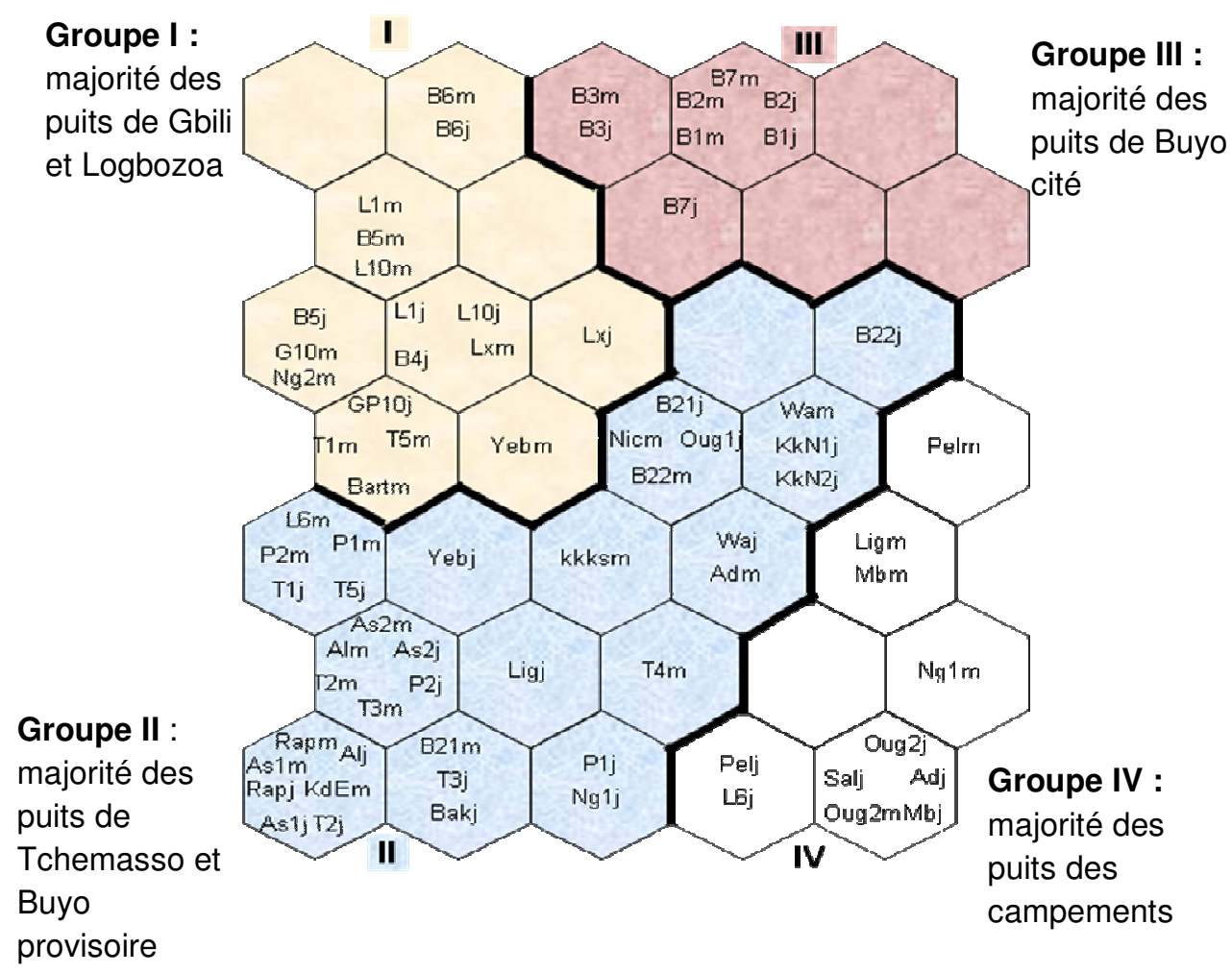

Figure 6 : Répartition des points d'échantillonnage sur la carte en fonction des paramètres physico-chimiques qui les discriminent.

Par ailleurs, les différents paramètres physicochimiques caractérisant les quatre groupes déjà indiqués sont représentés dans la figure 7 . Ainsi, les points d'eau du groupe I sont caractérisés par de fortes conductivités et des températures élevées avec des teneurs relativement élevées en éléments azotés $\left(\mathrm{NH}_{4}{ }^{+}, \mathrm{NO}_{2}{ }^{-}\right.$et $\left.\mathrm{NO}_{3}{ }^{-}\right)$. Les puits de ce groupe ont des profondeurs relativement faibles. Le groupe II se distingue par des profondeurs de puits élevées avec des eaux aux températures et aux conductivités relativement élevées. Les concentrations en éléments azotés sont faibles. Quant au groupe III, il se singularise par des valeurs élevées de température, de conductivité d'une part, et par de très fortes teneurs en éléments azotés et en cations majeurs d'autre part. Enfin le groupe IV est caractérisé par des profondeurs relativement élevées, de basses températures et de faibles conductivités. 

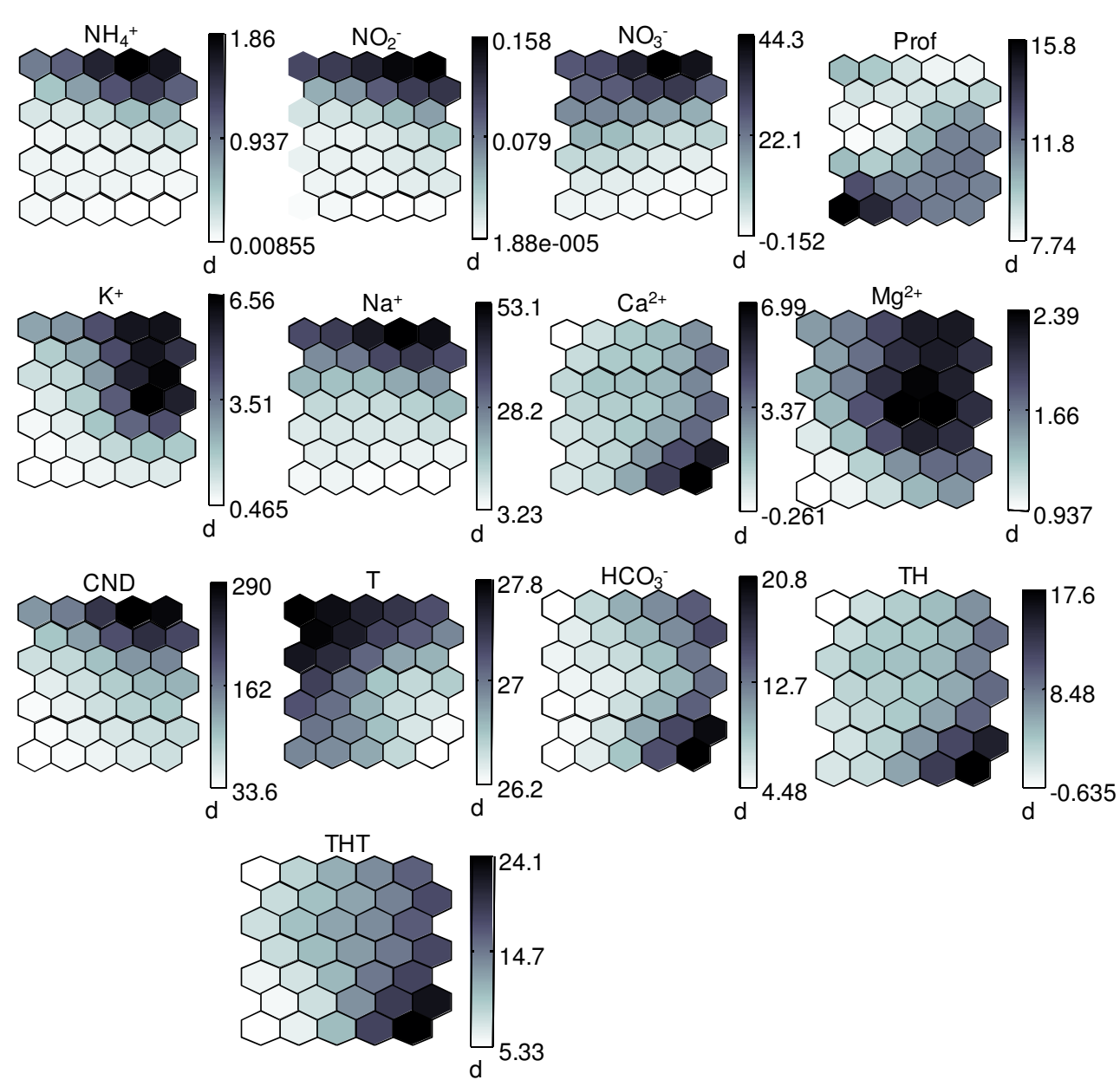

Figure 7 : Gradient de valeurs de chaque paramètre physico-chimique sur la carte de Kohonen ( $d=$ échelle ; couleur foncée $=$ valeurs fortes $;$ couleur claire $=$ valeurs faibles, Prof $=$ Profondeur, $C N D=$ Conductivité, $T=$ Température) .

\section{DISCUSSION}

La présente étude se propose de caractériser la qualité physico-chimique des eaux de puits à usage domestique sur le bassin versant de la Lobo, d'identifier l'origine de cette pollution et d'évaluer son impact sur la qualité des eaux. Les regroupements observés montrent que la conductivité, la température et les teneurs en éléments azotés sont plus élevés dans les puits peu profonds. Ces observations sont en accord avec celles faites par Hudak (1999) au Texas et Liu et al (2005). Les puits du groupe III montrent que dans le quartier de Buyo cité, la profondeur des puits n'est pas un critère très important car la plupart des puits, quelles que soient leurs profondeurs, ont des teneurs élevées en éléments azotés. Ainsi, aucune relation entre les concentrations en éléments azotés et la profondeur des puits n'est évidente dans ce quartier. Ces observations ont été également faites par Thorburn et al (2003) en Australie. Certains puits présentent des concentrations en ammonium supérieures à la norme de l'OMS $(0,5$ $\mathrm{mg} / \mathrm{L})$. Cela pourrait être dû à une mauvaise manipulation des puisettes par la population. Elles sont parfois déposées à même le sol comme l'ont remarqué Gelinas et al (1996). Cependant, les puits de Buyo cité présentent des teneurs plus élevées que celles des autres points d'eau. Ceci est sans doute à mettre en adéquation avec la forte densité de population observée dans ce quartier, au manque de salubrité, à l'absence de dispositifs d'assainissement approprié à la protection des eaux souterraines. En matière d'assainissement individuel, les équipements correspondent dans la grande majorité des cas à des latrines et puisards très mal entretenus et proches de la nappe (Chippaux et al 2002). Ainsi, une pollution d'origine domestique est à incriminer autour de ces points d'eau. Les concentrations en nitrite sont en général inférieures à la norme de l'OMS. Par contre, 
certains puits de la ville et des villages présentent des valeurs supérieures. Comme l'ammonium et le nitrite, les concentrations en nitrate sont aussi élevées dans certains puits et parfois où les teneurs en ammonium sont aussi élevées (spécifiquement les puits de Buyo cité avec des concentrations en nitrate supérieures à la norme de l'OMS (50 mg/L). Ceci est également en adéquation avec la pollution d'origine domestique liée à la forte densité de population observée autour de ces points d'eau. En général, les teneurs en éléments azotés sur le bassin versant sont faibles. Cependant,

\section{CONCLUSION}

L'étude révèle l'existence quasi généralisée des nitrates dans les eaux de puits. Elle montre que les puits des campements, en grande partie situés dans les plantations ont des eaux dont les teneurs sont très faibles en nitrate. Cependant, les eaux des puits des villages et de la ville, plus précisément celles de Buyo cité (quartier de densité de population élevée) ont des teneurs plus élevées. Cela démontre que l'agriculture, indexée dans la contamination en nitrate des eaux n'est pas la responsable. Ce sont les activités domestiques,

\section{REMERCIEMENT}

Nos remerciements vont à l'endroit du CRDI (Centre de Recherche pour le Développement International) à travers le financement d'un projet réalisé dans la région

\section{RÉFÉRENCES BIBLIOGRAPHIQUES}

Brosse S, Lek S, Dauba F, 1999a: Predicting fish distribution in a mesotrophic lake by hydroacoustic survey and artificial neural networks. Limnology and Oceanography, 44: pp 1293 - 1303.

Brosse S, Guegan J F, Tourenq J N, Lek S, 1999b: The use of artificial neural networks to assess fish abundance and spatial occupancy in the littoral zone of a mesotrophic lake. Ecological Modelling, 120: pp 299-311.

Brosse S, Dauba F, Oberdorff T, Lek S, 1999c: Influence of some topographical variables on the spatial distribution of lake fish during summer stratification. Archiv für Hydrobiologie, 145 : pp 359 - 371.

Brosse S, Giraudel J L, Lek S, 2001: The temporal dynamic of fish population assemblage and community structure. Ecological Modelling, 146 (1 - 3): pp 159 - 166.

Chippaux J P, Houssier S, Gross P, Bouvier C, Brissaud F, 2002: Étude de la pollution de des concentrations dans certains puits excèdent les normes de l'OMS en ce qui concerne l'eau de boisson. C'est particulièrement le cas des puits de la ville et plus précisément de ceux de Buyo cité. En effet, ce quartier est la partie du bassin la plus dense en population, suivi des deux villages Gbili et Logbozoa. Ainsi, les teneurs en éléments azotés, signes d'une contamination d'origine anthropique (pollution domestique) des eaux souterraines, sont plus élevées dans les zones à forte densité de population.

le défaut d'assainissement, les latrines mal installées compte tenu de l'exigüité des habitations, donc la densité de population qui contribuent à l'augmentation des teneurs en nitrate. Dans l'immédiat, une campagne de sensibilisation bien conçue sur l'assainissement et la santé publique devrait être initiée à l'endroit des populations pour permettre d'améliorer et de protéger la qualité de l'eau des puits traditionnels dans le bassin versant de la Lobo.

de Buyo et l'Université Nanguy Abrogoua (ex Université Abobo-Adjamé).

l'eau souterraine de la ville de Niamey, Niger. Bull Soc Pathol Exot, 94, 2, 119-123.

Faillat J P, 1990 : Origine des nitrates dans les nappes de fissures de la zone tropicale humide Exemple de la Côte d'Ivoire. J. Hydro., 113 : 231-264.

Gelinas Y, Randall H, Roubidoux L, Schmit J P, 1996: Well water survey in two districts of Conakry (Republic of Guinea), and comparison with the piped city water. Water Research, 30: pp 2017-2026.

Gevrey M, 2003: Modélisation de la diversité et de la structure des communautés aquatiques par la technique des réseaux de neurones artificiels. Thèse de Doctorat, Université Pierre et Marie Curie (Paris VI), France, 101p.

Giraudel J L et Lek S, 2001: A comparison of selforganizing map algorithm and some conventional statistical methods for ecological community ordination. Ecological Modelling, 146 (1 - 3): pp 329 - 339. 
Harris J, Loftis J C, Montgomery R H, 1987: Statistical methods for characterizing ground water quality. Ground Water, 25(2): pp 185-193.

Hudak P F, 1999: Chloride and nitrate distributions in the hickory aquifer, central texas, USA. Environment International, 25: pp 393-401.

Kohonen T, 1982: Self - Organized formation of topologically correct features maps. Biological Cybernetics, 43 : pp 59 - 69.

Kohonen T, 1995: Self - Organizing Maps. SpringerVerlag, Series in Informatique Sciences, 30, Heidelberg, 362p.

Laë R, Lek S, Moreau J, 1999: Predicting fish yield of African lakes using neural networks. Ecological Modelling, 120 : pp 325 - 335.

Lek S, Delacoste M, Baran P, Dimopoulos I, Lauga J, Aulagnier S, 1996: Application of neural networks to modelling nonlinear relationships in ecology. Ecological Modelling, 90: pp 39 52.

Lek S, Giraudel J L, Guégan J F, 2000: Neuronal networks: algorithms and architectures for ecologists and evolutionary ecologists. In Lek S. \& Guégan J. F. (Eds.). Artificial Neuronal Networks: Application to Ecology and Evolution. Springer - Verlag, Berlin : pp 3 - 27.

Liu A, Ming J, Ankumah R O, 2005: Nitrate contamination in private wells in rural Alabama, United States. Sci. Total Environ, 346: pp 112-120.

Mambo V, Tidou A S, Yapo O B, Houenou P 2001: Évaluation de l'état trophique du lac de Buyo (Côte d'Ivoire) : aspects physico-chimiques et biologiques, J.Soc. Ouest-Afr. Chim.; 011: pp 95-134

Ohou M J A, Mambo V, Yapo B O, M Seka A, Tidou A S, Kamagaté B, Houenou P V, 2008: Temporal and Spatial Variation of Nitrate Levels in Traditional Water -Supply Wells in the Area of Buyo, Côte d'Ivoire. Jounal of Applied Sciences 8 (18), pp 3096-3107

Pacheco J, Marin L, Cabrera A, Steinich B, Escolero O, 2001: Nitrate temporal and spatial patterns in 12 water supply wells, Yucatan, Mexico. Environmental Geology N ${ }^{\circ} 40$, pp 708-715.

Savané L, Goula-Bi T A, Aristide D G, Kouamé K L, 2005: Vulnerability assessment of the Abidjan quaternary aquifer using the DRASTIC method. Groundwater Pollution in Africa ; 115124.
Siegel S, 1956: Nonparametric Statistics for the Behavioral Sciences. McGraw Hill: New York ; 303.

Thorburn P J, Biggs J S, Weier K L, Keating B A, 2003: Nitrate in groundwaters of intensive agricultural areas in coastal Northeastern Australia. Agriculture, Ecosystems and Environment, 94: pp 49-58.

Tomassone R, Dervin C, Masson J P, 1993: Biométrie : Modélisation des Phénomènes Biologiques. Masson ; Paris; 1-553.

Tudesque L, Gevrey M, Grenouillet G, Lek S, 2008: Long-term changes in water physicochemistry in the Adour-Garonne hydrographic network during the last three decades. Water Research, 42: pp 732-742.

Yapo O B, Mambo V, Tidou A S, Houénou P V, 2008: Étude analytique des caractéristiques chimiques d'un lac eutrophe en milieu tropical : la conductivité comme indicateur de trophie du lac de Buyo (Côte d'Ivoire), J. Soc. Ouest-Afr. Chim. 025, pp 87-108.

Yapo O B, Mambo V, Séka A, Ohou M J A, Konan F, Gouzilé V, Tidou A S, Kouamé K V, Houénou $P$ V, 2010: Évaluation de la qualité des eaux de puits à usage domestique dans les quartiers défavorisés de quatre communes d'Abidjan (Côte d'Ivoire) : Koumassi, Marcory, Port-Bouet et Treichville. Int. J. Biol. Chem. Sci. 4(2): pp 289-307. 